\title{
eJRIEPS
}

Ejournal de la recherche sur l'intervention en éducation physique et sport

$36 \mid 2015$

Varia

\section{Corrélation entre orientation tactique du jeu et quantité d'activité physique en badminton}

Correlation between physical activity and tactically-oriented play in badminton

\section{Olivier Dieu}

\section{(2) OpenEdition}

Journals

Édition électronique

URL : http://journals.openedition.org/ejrieps/1481

DOI : 10.4000/ejrieps. 1481

ISSN : 2105-0821

Éditeur

ELLIADD

Référence électronique

Olivier Dieu, «Corrélation entre orientation tactique du jeu et quantité d'activité physique en badminton », eJRIEPS [En ligne], 36 | 2015, mis en ligne le 01 juillet 2015, consulté le 03 octobre 2019. URL : http://journals.openedition.org/ejrieps/1481; DOI : 10.4000/ejrieps.1481

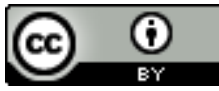

La revue eJRIEPS est mise à disposition selon les termes de la Creative Commons Attribution 4.0 International License. 
eJRIEPS 36 juillet 2015

\section{Corrélation entre orientation tactique du jeu et quantité d'activité physique en badminton}

Olivier Dieu

Université de Lille, URePSSS, EA 4110, Lille, France

Résumé

Le but de cette étude est de montrer que l'augmentation de la quantité d'activité physique en badminton n'est pas une fonction linéaire du niveau d'expertise du joueur mais qu'elle est corrélée à l'évolution de sa préoccupation tactique. 200 données ont été recueillies à partir des sets joués par une population de 99 badistes allant du débutant à l'expert. Les joueurs ont été classés en fonction de cinq étapes d' "expérience " en badminton au regard d'une classification conative. Les étapes 2 et 4 se caractérisent par une préoccupation tactique dans la mesure où la prévalence conative est davantage fonctionnelle que technique (étape 3) ou structurale (étape 1). L'activité physique a été mesurée par un accéléromètre. Les résultats montrent que la quantité d'activité physique (counts / sec) est comprise entre 115 (Etape 1) et 155 (Etape 5) et diffère significativement selon les étapes conatives. On note une forte évolution (+13\%) entre l'étape 1 et l'étape 2 , une régression de l'étape 2 à l'étape $3(-3 \%)$, une forte évolution entre l'étape 3 et l'étape $4(+22 \%)$ et enfin une légère augmentation en étape $5(+1 \%)$. L'évolution de la quantité d'activité physique en badminton, du débutant à l'expert, n'est donc pas linéaire. On note une inflexion significative de l'activité physique aux seuils des étapes 2 et 4, caractéristiques d'une mobilisation tactique du joueur. L'orientation tactique du jeu est donc corrélée à l'augmentation de la quantité de mouvement. En intervention, l'objectif de "mobilisation physique " des élèves ne saurait donc être atteint par des situations de déplacements décontextualisées et progressives mais par un respect du principe directeur de l'activité.

Mots clés: quantité d'activité physique- intention tactique - badminton- intervention responsable 


\section{eJRIEPS 36 juillet 2015}

\section{Introduction}

A l'heure où la thématique de la santé (re)devient prégnante dans le champ des STAPS, on assiste logiquement à une recrudescence des études privilégiant des méthodologies issues des sciences de la vie et les applications pédagogiques en découlant : rationnelles, contrôlées, progressives. Certes, la sédentarité (Inserm, 2008) comme l'obésité (Vigarello \& Guiet-Sylvain, 2011) sont un fléau de notre société hypermoderne (Lipovetsky, 1983) et force est de constater que les élèves aujourd'hui sont plus que jamais d'éternels débutants (Dieu, 2010).

Face à ce constat, certains pays d'Amérique du Nord comme le Québec ont clairement opté pour une éducation physique axée sur la dépense énergétique (Kino-Québec, 1999) qui préconise, dans une perspective de santé, que: «tous les enfants doivent pratiquer des activités physiques d'intensité moyenne et élevée trois fois ou plus par semaine pendant au moins vingt minutes par séance » (Kino-Québec, 2000).

Pour autant, l'intervention en activité physique, en France, saurait-elle se réduire à la mise en place d'un protocole progressif de mobilisation mécanique sur tapis roulant ? Doit-on revenir, en badminton, à des exercices fortement décontextualisés comme des déplacements à vide de type shadow (Laffaye, 2011) pour que les élèves débutants se mettent en action?

L'injonction de mouvement dans les pratiques pédagogiques est-elle efficace, voire même souhaitable? Les pratiques d'intervention, si elles souhaitent dépasser un certain déductivisme à l'égard des sciences de références (Léziart, 2010), ne doivent elle pas s'interroger en priorité sur les déterminants de la mobilisation mécanique en sport de raquette?

L'activité physique se définit comme «tout mouvement corporel produit par les muscles squelettiques qui entraîne une dépense énergétique supérieure à celle de repos " (US Department of Health an Human Services - National Center for Chronic Disease Prevention and Health Promotion, 1996). Elle peut donc se mesurer en quantité de mouvement (Caspersen, Powell, \& Christenson, 1985), via l'accélérométrie notamment (Chen \& Bassett, 2005).

Dans la littérature en sports de raquette, la mesure de cette activité physique des sujets n'est actuellement envisagée que d'une façon descriptive et ne concerne presque exclusivement que le haut niveau (Cabello Manrique \& Gonzales-Badillo, 2003, Hughes, 1994, Majumdar, et al., 1997 ; Faude, et al., 2007 ; Ooi, et al., 2009). 


\section{eJRIEPS 36 juillet 2015}

Peu d'études s'intéressent à l'activité physique du joueur dans une activité duelle en envisageant l'évolution de celle-ci ou alors elles mettent l'accent sur les facteurs extrinsèques à la pratique même comme les déterminants familiaux (Lauderdale \& al, 1997). D'autres travaux comparent l'évolution des novices et des experts mais ils ne concernent alors que les aspects décisionnels notamment l'acquisition des habiletés techniques indépendamment de l'activité physique (McPherson, 2000 ; Del Villar, et al., 2007).

Dès lors, la relation entre l'évolution de la dépense énergétique et l'évolution des aspects décisionnels (prévalence tactique du joueur) fait défaut dans la littérature.

Outre cette relative indépendance entre aspects énergétiques et aspects décisionnels, la littérature en sports de raquette situe ces deux aspects dans une progression linéaire corrélée avec le niveau d'expertise. Del Villar, et al. montrent par exemple, en tennis, que les experts « font des échanges plus longs que les novices pour marquer un point » (2007, p.360). Ce fait s'explique par le «niveau d'habileté et d'opposition plus élevé et la réduction des fautes non provoquées» (2007, p.362). La quantité de mouvement augmenterait donc bien avec le niveau d'expertise.

Par ailleurs les mêmes auteurs relaient l'idée, communément admise, selon laquelle le débutant joue en réaction alors que l'expert joue en intention, ce dernier ayant une capacité plus grande à prendre la bonne décision (Del Villar, et al., McPherson, 2000). A ce titre, les travaux de McPherson ont montré, dans de nombreuses activités telles que le baseball (McPherson, 1993a), le volley-ball (McPherson, 1993b), le tennis (McPherson, 1999a, 1999b) et à l'aide de méthodologies diverses: simulation vidéo (McPherson, 1993a) ou interrogation en jeu (McPherson, 1999a, 1999b) que les experts : " generated more tactical and associated conditions - actions concepts than novices ". A l'inverse, " novices made poor decisions, lacked sophisticated interpretations of conditions, and rarely planned actions»(McPherson, 2000, p.57). Le nombre des tactiques et leur complexité augmenterait donc bien avec le niveau d'expertise, les ouvrages actuels sur le badminton ne le réfutant jamais (Limouzin \& Wright, 2000 ; Laffaye, Rivas, \& Cabello Manrique, 2001).

Ainsi la littérature sur l'entraînement en sports de raquette quand elle compare l'activité des novices et des experts, à rapport de force équilibré, témoigne :

1. d'une relative indépendance entre aspects énergétiques et aspects décisionnels 


\section{eJRIEPS 36 juillet 2015}

2. d'une linéarité de ces deux aspects : la quantité de mouvement et la pensée tactique augmentent de manière linéaire avec le niveau d'expertise.

Les conséquences dans les pratiques s'expriment alors par les deux principes suivants :

- Ce serait la quantité de mouvement qui induirait la qualité du mouvement : "plus je m'entraîne, plus mon jeu va évoluer ».

- Ce serait le niveau de jeu qui conditionnerait l'apparition de la pensée tactique (aspects décisionnels en termes de plans d'actions).

La résultante, dans le sens commun, étant que "plus je pratique, plus je progresse en raquette et plus ma pensée devient tactique ". Cette croyance s'accorde bien avec les protocoles de type "science de la vie ", mécanistes et progressifs : l'évolution du joueur passant par une augmentation progressive et contrôlé de la quantité de mouvement.

Or, des auteurs comme Majumdar, et al. (1997) remettent en cause la progression linéaire de l'activité physique comme facteur de performance. Ils montrent que justement en badminton, l'entraînement, souvent trop intense au plan physiologique par rapport au match peut conduire à une altération de la performance, celle-ci étant basée sur la coordination dans ces sports d'habileté. La quantité d'activité physique peut donc nuire à l'efficacité particulièrement dans les sports à prédominance tactique. Cette idée suggère, en termes de méthodes d'entraînement, que c'est le jeu qui conditionne l'augmentation du mouvement et non l'inverse.

L'approche conative (Bui-Xuân, 1998) se fonde sur l'idée que l'intention tactique ou " fonctionnalité » n'augmente pas de manière linéaire avec le niveau de jeu. Pour cet auteur, la progression en activité sportive ne se résume pas à une simple augmentation du niveau de performance mais à un changement d'étape conative, c'est-à-dire un changement dans le sens de la mobilisation du joueur. Celui-ci mobilise, en fonction de son expérience (étape conative), de façon prévalente, soit sa structure (capacités physiques), soit sa technique (habiletés motrices), soit sa fonctionnalité (aspects décisionnels ou choix tactiques). Contrairement aux approches cognitivistes pré citées, déjà critiquées en leur temps par Evans (1990), qui assimilent la tactique à la verbalisation par le joueur de plans d'action (Kail \& Bisanz, 1982) et qui postulent que cette pensée tactique progresse de manière linéaire avec le niveau d'expertise et l'âge (McPherson \& Thomas, 1989), Bui-Xuân (1999) définit la tactique in situ comme une réorientation de la direction de l'action de jeu vers une prévalence davantage fonctionnelle que structurale ou technique. La décision résulte ici d'un couplage fonctionnel entre un individu et un contexte (Klein, 1997). 


\section{eJRIEPS 36 juillet 2015}

L'approche conative est basée sur une classification qui modélise d'une part le sens profond de l'activité, son "principe directeur ${ }^{1}$ " et d'autre part l'évolution du sens que chaque individu confère à son action en fonction de son expérience dans l'activité (le curriculum conatif). Le sens de l'activité, son principe directeur, représente la relation intime qui lie les trois dimensions: structurale, technique et fonctionnelle, la part de chacune par rapport aux autres, leurs proportions, leur contribution à l'exécution de la prestation » (Bui-Xuân, 1999, p.79). Par exemple le badminton, comme tous les sports de raquette (et d'opposition en général), est une activité à dominante clairement fonctionnelle, mais en proportion, elle est techniquement moins exigeante que le tennis mais structuralement plus exigeante que le tennis de table. Le sens du sujet évolue lui en fonction de son expérience de jeu à travers cinq étapes conatives du débutant à l'expert. Chaque étape se définit par la prévalence conative du joueur en fonction de son vécu dans l'activité (étape 1 : prévalence structurale, étape 2 : prévalence fonctionnelle, étape 3: prévalence technique, étape 4: prévalences technico-fonctionnelle, étape 5, d'expertise: prévalences structurales, fonctionnelles et techniques). Ce curriculum conatif et les critères de classification aux différentes étapes a fait l'objet d'utilisations dans des études récentes sur la pratique sportive (Vanhelst, et al., 2012a) et notamment en badminton (Dieu, 2012). Le tableau 1 les répertorie.

\footnotetext{
${ }^{1}$ Cette notion se rapproche de la notion d' « essence » qui « relève de la sphère émotionnelle ou encore de connotations socioculturelles qui accompagnent ou gouvernent la pratique de l'activité physique et sportive ou sa simple évocation » (Boda et Récopé, 1991). Bui-Xuân définit le principe directeur comme le sens originel des APSA : «ce pourquoi les pratiquants s'y sont adonnés au fil du temps »
} 


\section{eJRIEPS 36 juillet 2015}

Tableau I. Description du curriculum conatif et des indicateurs en Badminton

\begin{tabular}{|c|c|c|c|}
\hline Etapes conatives & Description du comportement & Indicateurs de classement & $\begin{array}{l}\text { Prévalence } \\
\text { tactique }\end{array}$ \\
\hline $\begin{array}{l}\text { Etape } 1 \\
\text { Structurale } \\
\text { Conation } \\
\text { Renvoyer }\end{array}$ & $\begin{array}{l}\text { Le joueur est relanceur, il } \\
\text { repousse le volant de l'autre côté } \\
\text { du filet. Le volant est pour lui un } \\
\text { danger dont il doit se débarrasser. }\end{array}$ & $\begin{array}{l}\text { Renvois longs axe central. Pas } \\
\text { de différenciation de statut } \\
\text { d'attaquant ou défenseur. }\end{array}$ & - \\
\hline $\begin{array}{l}\text { Etape } 2 \\
\text { Fonctionnelle } \\
\text { Conation } \\
\text { Diriger }\end{array}$ & $\begin{array}{l}\text { Le joueur est un placeur, il } \\
\text { cherche à diriger le Volant dans } \\
\text { l'espace libre. }\end{array}$ & $\begin{array}{l}\text { Variations de trajectoires et } \\
\text { apparition des statuts. Le } \\
\text { dominé court après le volant. Le } \\
\text { dominant tente d'envoyer le } \\
\text { volant du côté où l'adversaire } \\
\text { n'est pas. }\end{array}$ & ++ \\
\hline $\begin{array}{l}\text { Etape } 3 \\
\text { Technique } \\
\text { Conation = Placer } \\
\text { un coup gagnant }\end{array}$ & $\begin{array}{l}\text { Le joueur est un technicien } \\
\text { (smash, amorti, dégagement), qui } \\
\text { « se regarde dans l'exécution de } \\
\text { la frappe » et tarde donc à se } \\
\text { replacer. }\end{array}$ & $\begin{array}{l}\text { Dégagement ou Smash en } \\
\text { attaque. Temps d'arrêt dans le } \\
\text { replacement pour observer le } \\
\text { résultat de son action. }\end{array}$ & - \\
\hline $\begin{array}{l}\text { Etape } 4 \\
\text { Contextuelle } \\
\text { Conation } \\
\text { Enchaîner }\end{array}$ & $\begin{array}{l}\text { Le joueur est tacticien: il } \\
\text { enchaine les frappes dans une } \\
\text { séquence de jeu. II joue avec le } \\
\text { temps (intercepte en attaque / } \\
\text { relève le volant en défense) }\end{array}$ & $\begin{array}{l}\text { Replacement orienté après la } \\
\text { frappe (enchaînements) et } \\
\text { apparition du jeu en } \\
\text { interception. }\end{array}$ & +++ \\
\hline $\begin{array}{l}\text { Etape } 5 \\
\text { Expertise } \\
\text { Conation = } \\
\text { Imposer son style } \\
\text { de jeu }\end{array}$ & Le joueur est expert. & $\begin{array}{l}\text { Mobilise sa structure propre et } \\
\text { ses techniques selon son profil } \\
\text { de (« rameur », } \\
\text { « attaquant», «prendre le } \\
\text { filet »...) pour construire le point }\end{array}$ & +++ \\
\hline
\end{tabular}

A l'étape 1 (structurale), la question première du débutant est la suivante : comment se débarrasser du volant? Avant de «gagner », la préoccupation est de « ne pas perdre », l'intention du joueur est alors de repousser le plus fort possible le volant dans le cours adverse, c'est pourquoi les capacités physiques sont les premières mobilisées. A partir du 


\section{eJRIEPS 36 juillet 2015}

moment où le joueur a réglé le problème du renvoi, se pose alors la question suivante : comment battre l'adversaire? Dès lors il commence à jouer dans les espaces libres pour faire bouger son adversaire. A cette étape 2, dite fonctionnelle, les ressources décisionnelles sont prioritairement mobilisées. A l'étape 3 se pose la question de l'efficacité : comment font ceux qui gagnent? Le joueur entre dans une étape technique : il perd en fonctionnalité ce qu'il gagne en efficacité technique car il est préoccupé par le beau geste, l'exécution du mouvement. Ce n'est qu'à l'étape 4, contextuelle, que le joueur est capable de contextualiser la technique dans un enchaînement d'actions. A cette étape la prévalence tactique émerge à nouveau : la mobilisation du joueur est à la fois technique et fonctionnelle. Enfin, l'étape d'expertise (étape 5), se caractérise par une mobilisation optimale des ressources structurales, fonctionnelles et techniques du joueur pour fonder un style de jeu.

Bui-Xuân $(1998,1999)$ montre ainsi que la pensée tactique ou fonctionnalité est déjà caractéristique du niveau 2 (étape qualifiée de fonctionnelle), diminue en étape 3 (étape qualifiée de technique) pour redevenir prioritaire au niveau 4 (étape dite contextuelle). Cette idée n'est d'ailleurs pas en contradiction totale avec l'approche cognitiviste (Abernethy, Thomas, \& Thomas, 1993) qui ne nie pas l'activité tactique des novices, celleci étant seulement d'une autre nature : « les experts utilisent des stimuli différents de ceux des novices dans les situations de résolutions de problème en sport » (Abernethy cité par McPherson, 1993, p. 320).

Nous reprendrons donc à notre compte, à travers le modèle conatif (Bui-Xuân, 1999 ; Vanhelst, 2012), cette relation spécifique entre évolution du niveau de jeu et évolution de la pensée tactique (ou fonctionnalité) pour émettre l'hypothèse suivante :

l'activité physique, mesurée par un accéléromètre, diffère de manière significative en fonction des étapes conatives, notamment en étape 2 et 4 , où l'aspect tactique devient prévalent. »

\section{Méthode}

\subsection{Participants}

Quatre-vingt-dix neufs adolescents et adultes sains âgés de 16 à 43 ans ont participé à cette étude. Ils étaient tous volontaires et étaient soit étudiants, soit joueurs de badminton affiliés à la FFBa (62 hommes et 37 femmes). Leurs caractéristiques sont détaillées dans le tableau 1. 


\section{eJRIEPS 36 juillet 2015}

Tableau II. Caractéristiques des sujets.

\begin{tabular}{ccccccc}
\hline & Hommes & $\mathrm{M}$ & Ec & Femmes & $\mathrm{M}$ & Ec \\
\hline $\mathrm{N}$ & 62 & & & 37 & & \\
Age (années) & $16-43$ & 21,3 & $\pm 5,0$ & $20-34$ & 20,4 & $\pm 3,9$ \\
Taille $(\mathrm{cm})$ & $160-195$ & 178,3 & $\pm 6,4$ & $157-189$ & 166,1 & $\pm 6,2$ \\
Poids $(\mathrm{kg})$ & $53-102$ & 74,5 & $\pm 9,7$ & $44-90$ & 59,4 & $\pm 7,7$ \\
& & & & & & \\
\hline
\end{tabular}

Les sujets ont joué plusieurs sets contre des adversaires différents. Ils ont tous été filmés et classés par niveau d'expérience $(1,2,3,4,5)$ à l'aide des critères du curriculum conatif (tableau 1). Afin d'avoir des rapports de force homogènes, les données conservées ne pouvaient être retenues à l'avance. Ainsi c'est le score du set joué qui nous permettait de conserver ou non la donnée : pour qu'une donnée soit conservée il fallait que le score du perdant soit supérieur à 11 points.

\subsection{Outils}

2.2.1. Classification des sujets dans les étapes conatives

Pour « classer » nos sujets nous avons utilisé le curriculum conatif appliqué à la pratique sportive (Bui-Xuân, 1999) et plus récemment à la pratique du Badminton (Dieu, 2010). Celui-ci est résumé dans le tableau 1. La classification théorique des étapes conatives (tableau 1) a été validée grâce à un test vidéo proposé à dix spécialistes de badminton qui composait le jury du concours national de recrutement des professeurs d'éducation physique en France en 2011. Ce test interactif consistait en deux phases: une présentation vidéo de la classification conative en badminton (étapes conatives et indicateurs macroscopiques pour chaque étape) suivie d'une présentation de seize vidéos où les jurys devaient entourer l'étape correspondant à la vidéo et préciser l'indicateur correspondant. Le taux de validité du test est satisfaisant puisqu'il révèle une marge de $5 \%$ d'erreur pour des jurys d'âge, de région et donc de formation initiale très différentes. De plus, contrairement au jury qui n'a eu que six minutes de visionnage, nous avons fonctionné pour cette étude sur des observations longues, doublées d'enregistrements vidéo qui ont permis une ou plusieurs observations a posteriori pour écarter les vidéos qui pouvaient être litigieuses.

Lors des observations préalables, si les indicateurs macroscopiques étaient repérés nous classions l'individu à l'étape correspondante. Lors des tests, nous confrontions systématiquement deux joueurs de même étape. 


\section{eJRIEPS 36 juillet 2015}

\subsubsection{Mesure de l'activité physique}

Tous les participants ont porté un accéléromètre pendant un set de badminton (21 points en « tie-break » sans prolongation). La seule consigne était de jouer «comme en match » en portant une ceinture élastique sur laquelle est fixé l'accélérométre GT3X situé dans le bas du dos au niveau de la colonne vertébrale, au contact de la peau (Figure 1).
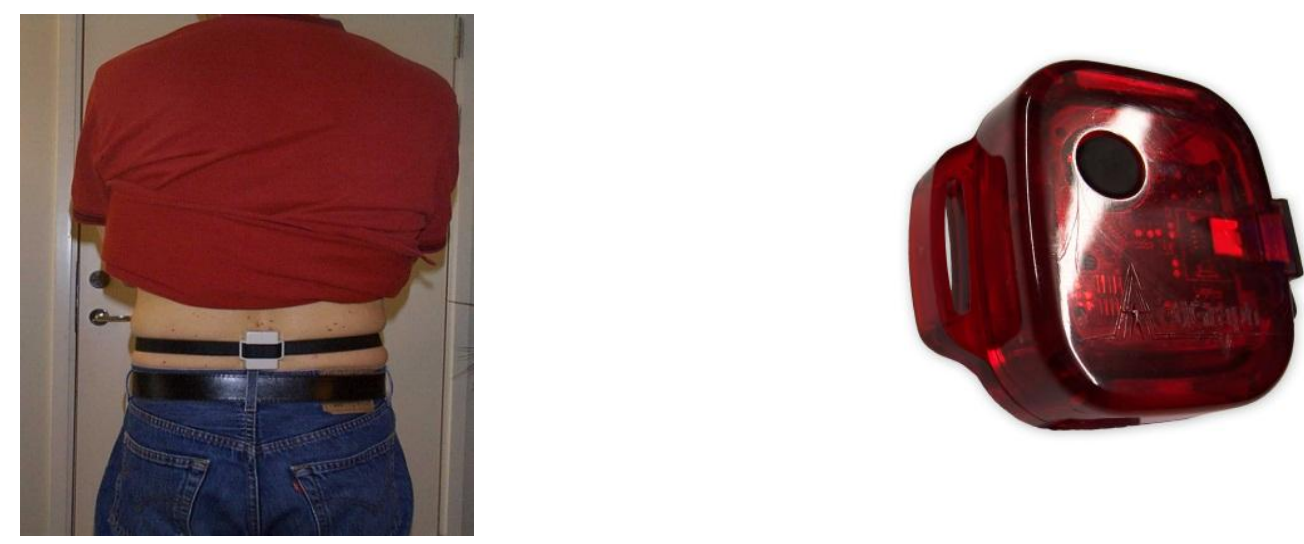

Figure 1. Pose de l'actimètre

Nous avons eu recours à l'accélérométrie (Bailey et al., 1995; Craig et al., 2003; De Jonge et al., 2007) pour quantifier l'activité physique car cet appareil a été l'objet de nombreuses validations de mesure d'activité physique contre des méthodologies de références comme la calorimétrie indirecte (Chen \& Bassett, 2005 ; Chu, McManus, \& Yu, 2007 ; Coleman, et al., 1997 ; Treuth, et al., 2004). Par ailleurs, les mesures obtenues par accéléromètre sont corrélées avec la consommation d'oxygène, marqueur de référence de l'activité physique (Rowlands, et al., 2004 ; Vanhelst, et al., 2010 ; Vanhelst, Béghin, Turck, \& Gottrand, 2011).

L'accélérométrie permet d'évaluer l'activité physique des sujets à partir de mesures mécaniques des mouvements (Chen \& Bassett, 2005). En effet, les mouvements du tronc et des membres induisent des accélérations qu'il est possible de mesurer à l'aide de capteurs. Les accélérations induites par le tronc sont proportionnelles à la force externe impliquée et reflètent donc le coût énergétique. Cette mesure repose sur un principe électrophysique : lors d'un déplacement, l'effet d'une accélération ou d'une décélération déforme les éléments d'une céramique piézo-électrique, ce qui donne naissance à une différence de potentiel. Cette impulsion électrique est proportionnelle à l'intensité des mouvements et enregistrable numériquement sous forme de fichier informatique. Les accélérations ou décélérations ainsi enregistrées rendent compte, de la fréquence, de la 


\section{eJRIEPS 36 juillet 2015}

durée et de l'intensité de l'effort physique produit par le corps en mouvement étudié (Leenders, et al., 2000). Les résultats sont exprimés en coups (counts).

Actuellement, l'accélérométrie représente la meilleure méthode de mesure objective et non invasive de l'activité physique. Ses principaux avantages sont sa précision, sa justesse et la possibilité de pouvoir l'utiliser dans les conditions habituelles de vie (Vanhelst, et al., 2009, 2012b, 2012c, 2012d). C'est donc une méthode de choix qui est applicable en recherche clinique ou épidémiologique pour des études de physiologie mais qui pourrait l'être aussi en pédagogie puisqu'elle permet une observation en condition réelle de jeu (Silva, et al., 2015).

De nombreuses études ont été faites sur la calibration de l'accéléromètre uni-axial (GT1M®, Pensacola, FL, USA) en laboratoire. En effet, l'accéléromètre GT1M a été calibré pour évaluer l'activité physique (sédentaire, légère, modérée, vigoureuse) et la dépense énergétique en fonction des accélérations mesurées par l'axe vertical de l'appareil (axe $\mathrm{X}$ ). Très récemment, le constructeur a développé un nouvel accéléromètre $(G T 3 X ®$, Pensacola, FL, USA) mesurant l'activité physique sur trois axes (vertical, antéropostérieur, et médio-latéral). La quantité d'activité physique est alors calculée grâce au vecteur magnitude (VM) qui est la racine carrée de la somme des counts enregistrés sur chaque vecteur ( $X, Y$ et $Z$ ). Nous avons récemment montré que les calibrations et déterminations des dépenses énergétiques réalisées précédemment avec l'accéléromètre uni-axial peuvent être rapportées au nouveau modèle GT3X (Vanhelst, et al., 2012c). II est également possible de convertir les données brutes de l'accéléromètre (VM) en dépense énergétique en utilisant des études de calibration (Eston, et al.,1998; Puyau, et al., 2002). Il a été démontré en laboratoire et dans les conditions habituelles de vie que les accéléromètres uniaxiaux étaient aussi performants que les accéléromètres triaxiaux dans l'évaluation de la DET quotidienne (Howe, et al., 2009 ; Vanhelst, et al., 2012d).

L'accéléromètre GT3X a été calibré selon les spécifications du constructeur et paramétré à la seconde.

\subsection{Protocole}

L'étude s'est déroulée sur une période allant du 13 janvier 2011 au 26 avril 2011. Au début du créneau d'entrainement dans lequel se déroulaient les mesures, les sujets repérés en amont comme appartenant à la même étape conative étaient mis au courant de la possibilité d'être mis à contribution pour une étude sur le badminton (pour ne pas affecter la validité interne de l'étude, la thématique de l'approche conative n'était pas exposée). 


\section{eJRIEPS 36 juillet 2015}

Les sujets volontaires se voyaient alors remettre un document rassemblant lettre d'information et fiche de consentement. Le protocole était le suivant :

- Jouer un set de badminton de 21 points sans prolongation, ni pause à la moitié du set, contre un adversaire imposé.

- Porter une ceinture accélérométrique sur laquelle est fixé l'actimétre GT3X (positionnement de l'appareil en bas du dos au niveau de la colonne vertébrale).

- Accepter de se faire filmer. Pour que l'influence de l'appareillage soit minime, le caméscope était placé en début de séance derrière un terrain afin que sa présence soit démystifiée.

Grâce à ce protocole et par le jeu des oppositions entre nos 99 sujets, nous avons pu recueillir 200 données (tableau 3 ).

Tableau III. Nombre de sets joués par étape conative

\begin{tabular}{lccc}
\hline & $\mathrm{N}$ & Homme: & Femme: \\
\hline Etape structurale & 40 & 9 & 31 \\
Etape fonctionnelle & 40 & 25 & 15 \\
Etape technique & 40 & 31 & 9 \\
Etape contextuelle & 40 & 32 & 8 \\
Etape d'expertise & 40 & 34 & 6 \\
\hline
\end{tabular}

\subsection{Analyses statistiques}

Toutes les données accélérométriques ont été téléchargées et analysées en utilisant "Statistical Package for the Social Science", Windows 11.5 (SPSS, Inc, Chicago, Illinois), et Excel 2003 (Microsoft, Inc, Redmond, Washington). On isole alors chaque set et on applique la formule de calcul du vecteur magnitude. La norme du vecteur magnitude (VM), exprimée par la formule suivante : $V M=\sqrt{ } x^{2}+y^{2}+z^{2}$, nous renseigne sur la quantité de mouvement ou dépense énergétique en counts par seconde.

Des analyses de variance (ANOVA) ont été faites pour comparer l'activité physique en fonction des étapes conatives afin de voir si les différences de moyennes entre les étapes étaient significatives. $(p<0,05)$. 


\section{eJRIEPS 36 juillet 2015}

\section{Résultats}

Le traitement des données actimétriques nous donne une moyenne de « counts » sur les trois axes à la seconde ainsi que la moyenne de la résultante sur les trois axes, appelée vecteur magnitude (notée VM, Cf. Table IV). On note (cf. Figure II) une forte évolution (+ $13 \%)$ de l'étape structurale à l'étape fonctionnelle (114,7 counts $\pm 13,2$ à 129,7 counts \pm $14,4)$ et ensuite une légère régression de $3 \%$ à l'étape technique $(129,7$ counts $\pm 14,4$ à $125,5$ counts $\pm 11,7)$. On note une augmentation nette $(+22 \%)$, à nouveau de l'étape technique à l'étape contextuelle (125,5 counts $\pm 11,7$ à 153,1 counts $\pm 21,2)$ puis une très légère augmentation $(1 \%)$ de l'étape contextuelle à l'étape d'expertise $(153,1$ counts \pm $21,2$ à 154,7 counts $\pm 9,9)$.

Tableau IV. Moyennes en counts pour chaque étape conative

\begin{tabular}{|c|c|c|c|c|c|c|c|c|c|}
\hline \multicolumn{2}{|c|}{$\begin{array}{c}\text { Axes de } \\
\text { l'accélérométre }\end{array}$} & \multirow{2}{*}{$\begin{array}{c}\text { Etape } 1 \\
67,1\end{array}$} & \multirow{2}{*}{$\begin{array}{c}\text { Etape } 2 \\
76,2\end{array}$} & \multirow{2}{*}{$\begin{array}{c}\text { Etape } 3 \\
74,7\end{array}$} & \multirow{2}{*}{$\begin{array}{c}\text { Etape } 4 \\
97,9\end{array}$} & \multirow{2}{*}{$\begin{array}{c}\text { Etape } 5 \\
92,0\end{array}$} & \multirow[t]{2}{*}{$F$} & \multirow[t]{2}{*}{$p$} & \multirow[t]{2}{*}{$\eta^{2}$} \\
\hline Axe $X$ & $M$ & & & & & & & & \\
\hline & SD & 11,7 & 11,0 & 9,1 & 18,9 & 8,5 & 9,26 & $<0,01$ & 0,93 \\
\hline \multirow[t]{2}{*}{ Axe $Y$} & $M$ & 56,1 & 61,8 & 61,0 & 71,9 & 79,7 & & & \\
\hline & SD & 10,7 & 10,9 & 9,3 & 13,0 & 8,2 & 8,16 & $<0,05$ & 0,90 \\
\hline \multirow[t]{2}{*}{ Axe Z } & $M$ & 60,0 & 70,0 & 66,2 & 76,4 & 77,9 & & & \\
\hline & SD & 8,0 & 8,1 & 7,4 & 9,0 & 6,8 & 9,78 & $<0,05$ & 0,91 \\
\hline \multirow[t]{2}{*}{ VM } & $M$ & 114,7 & 129,7 & 125,5 & 153,1 & 154,7 & & & \\
\hline & SD & 13,2 & 14,4 & 11,7 & 21,2 & 9,9 & 9,89 & $<0,001$ & 0,95 \\
\hline $\begin{array}{r}\text { Note : } \\
z^{2},\end{array}$ & orme & vecteur & gnitude & $\begin{array}{l}\text { प), expr } \\
\text { nouvem } \\
\text { secon }\end{array}$ & par la f & $\begin{array}{l}\text { nule suiv } \\
\text { énergét }\end{array}$ & te : & $\begin{array}{l}M=\sqrt{x^{2}} \\
\text { counts } p\end{array}$ & \\
\hline
\end{tabular}




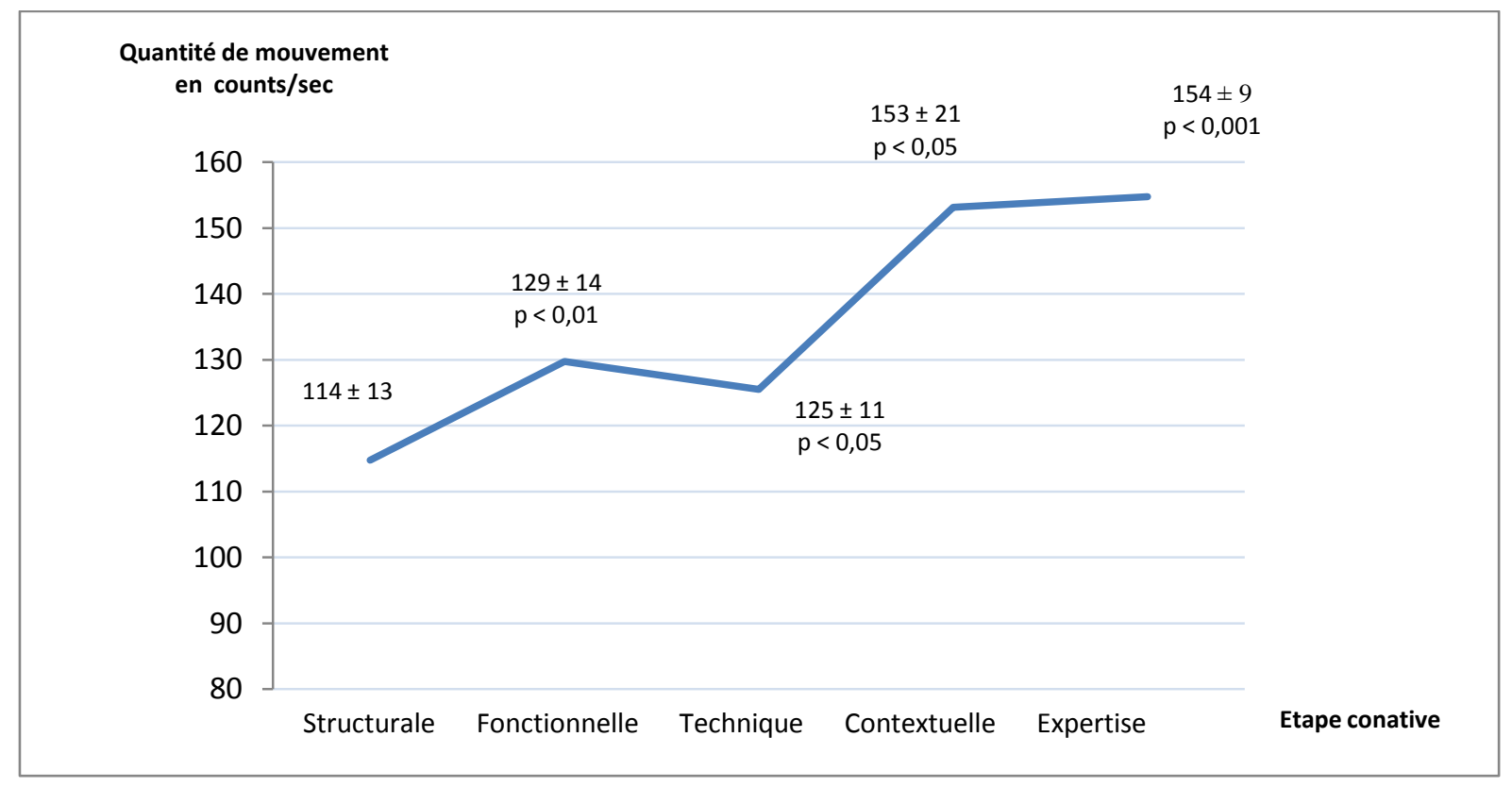

Figure 2. Quantité de mouvement (VM en counts /sec) par étape conative.

\section{Discussion}

Traditionnellement les études sur la dépense énergétique en sports de raquette se limitent au haut niveau (Cabello Manrique \& Gonzales Badillo, 2003 ; Carlson, Tyrell, \& Naughton, 1985 ; Ooi, et al., 2009) et utilisent des méthodologies lourdes et invasives comme les mesures de taux de lactate sanguin (Gosh, et al., 1990, Gosh, Goswami, \& Ahuja, 1993; Novas, Rowbottom, \& Jenkins, 2003). Elles aboutissent à des résultats génériques et généraux qui renforcent l'idée d'une évolution linéaire du débutant à l'expert. La présente étude est une des premières à s'intéresser à l'évolution de l'activité physique du débutant à l'expert dans l'activité badminton en utilisant l'actimétrie. Cet outil, facile à porter, permet de travailler sur des échantillons beaucoup plus grands et hétérogènes autorisant une étude in vivo. De plus en mettant en relation l'actimétrie avec l'évolution des intentions du joueur au cours de sa progression, l'actimétre apparaît comme un indicateur de corps mobilisé (Dieu, 2012).

Dans la littérature en sports de raquette, on note une relative indépendance entre aspects énergétiques et aspects décisionnels. Finalement, il est communément admis que l'activité physique (Del Villar, et al., 2007) tout comme l'activité décisionnelle (McPherson, 1993, 1999, 2000) augmente de manière linéaire avec le niveau d'expertise. En utilisant le modèle conatif matérialisant l'expérience en badminton sous formes d'étapes conatives, nous nous inscrivons en rupture avec ces modèles traditionnels. Cette approche, dans laquelle les changements d'étape conative se traduisent par une réorganisation des 


\section{eJRIEPS 36 juillet 2015}

prévalences structurales, fonctionnelles ou techniques en fonction du sens de la mobilisation du joueur, permet d'envisager une description plus qualitative des modifications dans les quantités de mouvement investies du débutant à l'expert. Ainsi, nos résultats suggèrent qu'en fonction des étapes conatives, la quantité de mouvement des badistes évolue de manière non progressive et non linéaire.

A l'étape 1 le joueur a une prévalence structurale : il se déplace peu et mobilise tout son corps dans le renvoi du volant car sa préoccupation est de le repousser fort dans le camp adverse. En étape 2, la prévalence devient fonctionnelle : le joueur commence à diriger le volant dans l'espace libre même si ses techniques de frappe restent encore primaires (par exemple le joueur pousse le volant, en conservant l'alignement œil-tamis-volant, à droite pour jouer ensuite à gauche). Cela provoque de longues courses après le volant chez le joueur dominé qui se traduisent par une augmentation de l'activité physique. A l'étape 3 la prévalence fonctionnelle diminue au profit de la prévalence technique : le joueur est centré sur l'exécution de sa frappe. II en résulte une attitude plus statique qui se traduit par une stagnation de l'activité physique. A l'étape 4, la prévalence est technico-fonctionnelle. Le joueur contextualise ses techniques. II enchaîne ses frappes pour prendre son adversaire de vitesse (par exemple il saute pour intercepter et enchaîne avec un jaillissement vers le filet). II utilise de plus en plus le temps pour mettre son adversaire en défaut. II est dans une logique spatio-temporelle qui se traduit par une mobilisation dynamique. A nouveau à cette étape on note une forte augmentation de l'activité physique. En étape 5, structure, fonction et technique se rééquilibrent ce qui se traduit par une stagnation de l'activité physique.

L'étude de l'activité physique nous montre que les étapes 2 et 4 du curriculum conatif se caractérisent par une forte augmentation de l'activité physique du joueur. Ces étapes dites «fonctionnelle » et «contextuelle» se caractérisent par une orientation de jeu plus tactique (prévalence fonctionnelle) même si celle-ci n'est pas de même nature en fonction de ces étapes (spatiale en étape 2, spatio-temporelle en étape 4). C'est donc dans les étapes en consonance avec le principe directeur (Bui- Xuan, 1998) de l'activité badminton que la mobilisation du joueur est optimale. En effet, le badminton est une activité de score dans laquelle le joueur qui gagne n'est pas forcément le plus fort structuralement (comme en athlétisme), ni celui qui a la meilleure technique (comme en gymnastique) mais celui qui se joue de son adversaire (fonctionnalité tactique).

La quantité de mouvement n'est donc pas une loi puissance (proportionnelle) du niveau de jeu. 


\section{eJRIEPS 36 juillet 2015}

II y a donc bien des modifications qualitatives profondes de l'activité du joueur dans son curriculum conditionnées par le sens qu'il confère à son action (Bui-Xuân, 1998). Ceci nous permet de répondre à notre hypothèse: en badminton, l'activité physique est fonction non pas du niveau d'expertise mais de la modification de l'orientation de jeu vers une plus grande mobilisation fonctionnelle (ou prévalence tactique).

Ainsi, ce n'est pas le niveau d'expertise mais la fonctionnalité du jeu qui est le déterminant intrinsèque de la quantité de mouvements dans l'activité badminton. C'est donc davantage en orientant les propositions didactiques vers le principe directeur (score) de l'activité qu'en proposant des situations artificielles de déplacement (Laffaye, 2011), aussi progressives soient-elles, que l'on pourra « remettre les jeunes en mouvement ». Ainsi, respecter la spécificité décisionnelle des activités de raquette contribue à l'atteinte d'un autre objectif assigné à l'éducation physique, celui de la dépense énergétique. Dès lors, chaque situation visant à «mettre en mouvement les élèves », doit être saturée en fonctionnalité : il doit y avoir un adversaire, un score, une réversibilité des rôles et des alternatives, autant de variables qui constituent I'ADN (rapport jury CAPEPS, 2011) de la compétence propre $n^{\circ} 4$ des programmes d'EPS. L'échauffement, dans ce cadre, ne saurait être trop longtemps décontextualisé sous peine de transformer l'activité du sujet en athlétisme avec volant !

Les perspectives pratiques suggérées par nos résultats s'inscrivent en rupture au regard des méthodes d'intervention traditionnelles en badminton. Trop souvent, les enseignants, en voulant privilégier l'aspect décisionnel du badminton, réduisent l'activité physique des élèves en leur faisant verbaliser leurs stratégies ou remplir des fiches sur leur projet de jeu. Si nous ne contestons pas l'intérêt pédagogique des doublettes coach-joueur (Mascret, 2009), leur utilisation systématique réduit sensiblement le temps moteur, ce qui peut être préjudiciable à l'émergence in situ de la fonctionnalité, les élèves peinant à reproduire ce qu'ils «déclarent» en situation réelle de jeu. A l'inverse, certains enseignants, se disant experts, réduisent l'activité fonctionnelle des élèves en important des modèles issus de l'entraînement à l'école comme le shadow qui consiste à effectuer des gammes de déplacements en pas chassés avec simulations de frappes sans volant. Enfin, dans la majorité des cas, en badminton, on fait des matchs en montante descendante sur demi-terrain afin de gérer l'effectif.

En EPS, où la majorité des élèves est en étape fonctionnelle, le rôle de l'enseignant, soucieux de s'inscrire à la fois dans la spécificité tactique du badminton tout en développant les ressources physiques, est de proposer des dispositifs en consonance 


\section{eJRIEPS 36 juillet 2015}

ergo conative (Dieu, 2012) favorisant la fonctionnalité spatiale des joueurs. Nous définissons la consonance ergo-conative comme le respect par l'enseignant de la corrélation qui existe entre le sens mécanique du mouvement (direction spatiale de l'action de jeu, temporalité du jeu) et le sens que le joueur attribue à son action en termes de conations (Dieu, 2012). Chaque sujet, en fonction de son expérience, redéfinit le contexte de jeu, il n'y a pas un terrain de jeu (une scène prescrite) qui serait la même pour tous les badistes mais autant de « contextes altérés par l'action» (Dieu, 2012) à partir du moment où le joueur entre en scène. Ainsi un dispositif pédagogique ergo-conatif permet aux conations du joueur de s'exprimer car il respecte le sens de la mobilisation du joueur à l'étape où il se situe.

Ainsi à l'étape 2, augmenter la largeur des terrains, conserver l'opposition, donner une importance au score et à son évolution induira de facto de nombreux déplacements. Demander aux élèves d'aller toucher un plot entre chaque frappe sur un demi-terrain ne saurait avoir grand sens à cette étape! Limiter les matchs à la traditionnelle « montante descendante » (Descamps, 2008) sur des demi-terrains ne saurait non plus être d'une grande utilité car sur cette surface les joueurs n'ont pas les moyens techniques pour faire bouger leur adversaire. A l'étape fonctionnelle c'est l'augmentation de la largeur des terrains, donc de l'espace qui va faire émerger la fonctionnalité naissante car dans ce contexte le joueur a les moyens de faire bouger son adversaire en latéral sans grande technique (simple orientation du tamis), ce qui n'est pas le cas sur des demi terrains en profondeur ou la maîtrise du dégagement et de l'amorti de fond de court est nécessaire... A l'entraînement, le passage de l'étape technique à l'étape contextuelle ne saurait advenir par une augmentation de la charge physique via des routines imposées par l'entraîneur. La fonctionnalité temporelle qui donne le sens à l'enchaînement des frappes est ici occultée et la lassitude ne tardera pas à grandir à mesure que les fautes directes augmenteront au fil des séries. C'est justement la compréhension en acte de l'enchaînement frappe / replacement dans une perspective temporelle (quand dois-je sauter pour intercepter? après ce coup où dois je me replacer dans mon demi-terrain ?) qui va amener le joueur à répéter les séries car elles prendront du sens. Ici encore pour que le joueur se dépense plus, il doit comprendre en acte cette notion d'enchaînement. Ceci n'est possible que dans un contexte d'opposition ou semi-opposition (c'est-à-dire que le relanceur est cantonné à un rôle ou à une zone précise de jeu) où l'alternative est possible (par exemple, le relanceur distribue du filet alternativement : un long, un court. Dans cette situation, le coup long peut être : 


\section{eJRIEPS 36 juillet 2015}

- très haut, ce qui laisse au joueur qui travaille le temps d'effectuer sa rotation centrale pour smasher

- tendu, ce qui diminue le temps disponible et oblige le joueur qui travaille à rester de face, jaillir et intercepter pour smasher).

Nos résultats doivent néanmoins être nuancés. En effet, la distinction d'âge et de sexe en tant que variables influençant la quantité de mouvement n'a pas été retenue. Or certains auteurs ont montré que le sexe avait une influence sur l'intensité du match en tennis notamment (Fernandez, Mendez-Villanueva, \& Pluim, 2006).

\section{Conclusion}

Cette étude portait sur 99 joueurs de badminton de tous niveaux qui se sont opposés en contexte de match équipés d'un accéléromètre triaxial. Ces joueurs ont été classés en fonction de leur expérience du badminton dans une des cinq étapes du curriculum conatif. Dans le cadre du modèle conatif (Bui-Xuân, 1998 ; Vanhelst, 2012a) qui postule une relation spécifique entre évolution du niveau de jeu et évolution de la pensée tactique (ou fonctionnalité) l'hypothèse suivante a pu être vérifiée:

L'activité physique, mesurée par un accéléromètre, diffère de manière significative en fonction des étapes conatives, notamment en étape 2 et 4 , où l'aspect tactique devient prévalent.

Ainsi, ce n'est pas le niveau d'expertise mais la fonctionnalité du jeu (ou prévalence tactique) qui modifie quantitativement l'activité physique du joueur de badminton au cours de sa progression. Ces résultats suggèrent de se focaliser davantage sur le sens que le joueur attribue au jeu en fonction de son expérience plutôt que sur une simple augmentation de son activité physique en termes de déplacements sur le terrain. A quoi bon, en effet, demander à un sujet de bouger plus que ce que le jeu exige dans l'étape où il se situe?

A l'école, les propositions pédagogiques qui consistent en une simple augmentation de la quantité de mouvement du joueur devraient être abandonnées au profit de stratégies d'intervention plus responsables qui visent à aménager le milieu dans le sens de la mobilisation du sujet, c'est à dire dans le sens qu'il attribue au jeu en fonction de son étape conative.

A l'entraînement l'injonction de mouvement n'est pas plus souhaitable puisque c'est le jeu qui produit la mobilisation. Ainsi il serait préférable de ne pas dissocier la tactique de 


\section{eJRIEPS 36 juillet 2015}

l'entraînement physique car l'expérience du joueur en badminton témoigne d'une interrelation entre ces deux aspects.

Dans nos recherches futures, l'accélérométrie sera à nouveau utilisée pour investiguer les spécificités ergonomiques de chaque étape conative: nous avons déjà travaillé sur l'aspect spatial de la mobilisation en termes de direction de l'action de jeu (Dieu, 2012), nous allons prochainement nous intéresser aux temporalités en fonction des prévalences conatives, notamment à la façon dont le joueur gère l'alternance jeu / non-jeu au cours du set et en fonction du rapport de force.

\section{Bibliographie}

Abenerthy, B., Thomas, K. \& Thomas, J. (1993). Strategies for improving understanding of motor expertise (or mistakes we have made and things we have learned!). In J.L. Starkes \& F. Allard (Eds.), Cognitive issues in motor expertise (pp. 317-356). Amsterdam: Elsevier.

Bailey, R. C., Olson J., Pepper S. L., Porszasz J., Barstow T. J., Cooper D. M. (1995). The level and tempo of children's physical activities: an observational study. Medicine and Science in Sports and Exercise, 27(7), 1033-41.

Boda, B., \& Récopé, M. (1991). Instruments d'analyse et de traitement des APS à des fins d'enseignement, EPS, 231, 56-58.

Bui-Xuân, G. (1998). Le corps mobilisé, Montpellier, HDR : Université de Montpellier 1.

Bui-Xuan G. (1999). Modeling process teaching: Teaching physical education. In G.BuiXuân \& J Gleyse (Eds), Teaching physical education (pp. 79-96). Clermont Ferrand, France: AFRAPS.

Cabello Manrique D., Gonzalez-Badillo J.-J. (2003). Analysis of the Caracteristics of Competitive Badminton. British Journal of Sport and Medicine 37(1), 62-6.

Carlson, J., Tyrell, J., \& Naughton, G. (1985).Physiological responses during badminton games by elite Australian players. Badminton Sitelines, 13, 17-20.

Caspersen, C. J., Powell, K. E., Christenson, G. M. (1985). Physical activity, exercise and physical fitness: Definitions and distinctions for health-related research. Public Health Reports, 110, 126-131.

Chen, K. Y., \& Bassett, D. R., Jr. (2005). The technology of accelerometry-based activity monitors: Current and future. Medicine and Science in Sports and Exercise, 37, 490-500. 


\section{eJRIEPS 36 juillet 2015}

Chu, E. Y., McManus, A. M., \& Yu, C. C. (2007). Calibration of the RT3 accelerometer for ambulation and non ambulation in children. Medicine and Science in Sports and Exercise, 39, 2085-2091.

Coleman, K. J., Saelens, B. E., Wiedrich-Smith, M. D., Finn, J.D., \& Epstein, L. H. (1997). Relationships between TriTrac-R3D vectors, heart rate, and self-report in obese children. Medicine and Science in Sports and Exercise, 29, 1535-1542.

Craig, C. L., Marshall, A. L., Sjostrom, M, Bauman, A. E., Booth, M. L., Ainsworth, B. E., Pratt, M., Ekelund, U., Yngve, A., Sallis, J. F., Oja, P. (2003). International physical activity questionnaire: 12-country reliability and validity. Medicine and Science in Sports and Exercise, 35, 1381-1395.

De Jonge, L., DeLany, J. P., Nguyen, T., Howard, J., Hadley, E. C., Redman, L. M., Ravussin, E. (2007). Validation study of energy expenditure and intake during calorie restriction using doubly labelled water and changes in body composition. The American Journal of Clinical Nutrition, 85, 73-9.

Del Villar F., García González L., Iglesias D., Perla Moreno M., Cervelló E. M. (2007). Expert-novice differences in cognitive and execution skills during tennis competition. Perceptual and Motor Skills, 104(2), 355-65.

Descamps, O. (2008). Badminton : la montante descendante : situation de référence et/ou d'apprentissage. EPS, 332, 39-42.

Dieu, O. (2010). Badminton : l'éternel débutant. EPS, 343, 10-13.

Dieu, O. (2012). Expérience corporelle et sens du mouvement : matérialisation via l'actimétrie du "contexte altéré par l'action " dans l'évolution du joueur de badminton. STAPS, 98, 49-65.

Eston, R. G, Rowlands, A. V, Ingledew, D. K. (1998). Validity of heart rate, pedometry, and accelerometry for predicting the energy cost of children's activities. Journal of Applied Physiology, 84, 362-371.

Evans, J. (1990). Verbal reports of cognitive strategies: a note on Marques and Pereira. The Quartely Journal of Experimental Psychology, 42, 169-170.

Faude, O., Meyer, T., Rosenberger, F., Fries, M., Huber, G., Kindermann, W. (2007). Physiological characteristics of badminton match play. European Journal of Applied Physiology, 100(4), 479-85.

Fernandez, J., Mendez-Villanueva, A. Pluim, B. M. (2006). Intensity of tennis match play. British Journal of Sports and Medicine, 40(5), 387-91. 


\section{eJRIEPS 36 juillet 2015}

Ghosh, A. K., Majumbar, P., Goswami, A., Ahuja, A., \& Puri, T. P.S. (1990). Heart rate and blood lactate response in competitive badminton. Annals sport medicine, 5(2), 8588.

Ghosh, A. K., Goswami, A., \& Ahuja, A. (1993). Evaluation of a sports specific training programme in badminton players. Indian Journal of Medical Research, 98, 232236.

Howe, C. A., Staudenmayer, J. W., Freedson, P. S. (2009). Accelerometer prediction of energy expenditure: vector magnitude versus vertical axis. Medicine and Science in Sports and Exercise, 41, 2199-2206.

Hughes M. G. (1994). Physiological demands of training in elite badminton players. Science and racket sports, 33-37.

Kail, R. V., Bisanz, J. (1982). Cognitive strategies. In C.R. Puff (Ed.), Handbook of research methods in human memory and cognition (pp. 229-255). New York: Academic Press.

Kino-Québec (2000). L'activité physique, déterminants de la santé des jeunes, Synthèse du comité scientifique de Kino-Québec et applications. Gouvernement du Québec, Ministère de l'éducation, du loisir et du sport, 24 pages.

Kino-Québec (1999). Quantité d'activité physique requise pour en retirer des bénéfices pour la santé, Synthèse du comité scientifique de Kino-Québec et applications. Gouvernement du Québec, Ministère de l'éducation, du loisir et du sport, 16 pages.

Laffaye G., Rivas, F., \& Cabello Manrique, D. (2001). The role of visual information in the training of badminton players: informative currila. IV IBF World Coaches Conference. Seville, Spain, May 31-June 2.

Laffaye, G. (2011). Comprendre et progresser en badminton. Magny-Les-Hameaux, France : Chiron.

Lauderdale, D. S., Fabsitz, R., Meyer, J. M., Sholinsky, P., Ramakrishnan, V., Goldberg, J. (1997). Familial determinants of moderate and intense physical activity: a twin study. Medicine and Science in Sports and Exercise, 29(8), 1062-1068.

Leenders, N. Y. J. M., Sherman, W. M., Nagaraja, H. N. (2000). Comparisons of four methods of estimating physical activity in adult women. Medicine and Science in Sports and Exercise, 32, 1320-1326.

Leziart, Y. (2010). Les rapports entre les savoirs théoriques et les savoirs pratiques: pour un dépassement des rapports établis. eJRIEPS, 20, 48-63. 


\section{eJRIEPS 36 juillet 2015}

Limouzin, P, Wright, I. (2000). Badminton, vers le haut niveau. Paris : INSEP.

Lipovetsky, G. (1983). L'ère du vide : essais sur l'individualisme contemporain. Paris : Gallimard.

Majumdar, P., Khanna, G. L., Malik, V., Sachdeva, S., Arif, M., Mandal, M. (1997). Physiological analysis to quantify training load in badminton. British Journal of Sports Medicine, 31(4), 342-5.

Mascret, N. (2009). Les interactions "joueur-coach" en badminton et leur impact sur les apprentissages des élèves difficiles. eJRIEPS, 16, 55-72.

Mc Pherson, S. L. (1993a). The influence of player experience on problem solving during batting preparation in baseball. Journal of sport and exercise psychology, 15, 304325.

Mc Pherson, S. L. (1993b). Knowledge representation and decision making in sport. In J.L. Starkes \& F. Allard (Eds.), Cognitive issues in motor expertise (pp. 159-188). Armsterdam: Elsevier.

Mc Pherson, S. L. (1999a). Expert-novice differences in performance skills and problem representations of youth and adults during tennis competition. Research Quartely for Exercise and Sport, 70, 233-251.

Mc Pherson, S. L. (1999b). Tactical differences in problem representations and solutions in collegiate varsity and beginner women tennis players. Research Quartely for Exercise and Sport, 70, 369-384.

Mc Pherson, S. L. (2000). Expert-novice differences in planning strategies during collegiate singles tennis competition. Journal of sport and exercise psychology, 22, 39-62.

Mc Pherson, S. L., \& Thomas J. R. (1989). Relation of knowledge and performance in boys' tennis: age and expertise. Journal of experimental child psychology, 48, 190211.

Novas, A. M., Rowbottom, D. G., Jenkins, D. G. (2003). A practical method of estimating energy expenditure during tennis play. School of Human Movement Studies, Journal of Science and Medicine in Sport, 6(1), 40-50.

Ooi Ch., Tan, A., Ahmad, A., Kwong, K. W., Sompong, R., Ghazali, K. A., Liew, S. L., Chai, W. J., Thompson, M. W. (2009). Physiological characteristics of elite and sub-elite badminton players. Journal of Sports Sciences, 27(14), 1591-1599.

Puyau, M. R., Adolph, A. L., Vohra, F. A., Butte, N. F. (2002). Validation and calibration of physical activity monitors in children. Obesity Research, 10, 150-157. 


\section{eJRIEPS 36 juillet 2015}

Rowlands, A. V., Thomas, W. M., Eston, R. G., et al. (2004). Validation of the RT3 Triaxial accelerometer for the assessment of physical activity. Medicine and Science in Sports and Exercise, 36, 518-524.

Silva, P., Santiago, C., Reis, L. P., Sousa, A., Mota, J., \& Welk, G. (2015). Assessing physical activity intensity by video analysis. Physiological Measurement, 36, 10371046.

Treuth, M. S., Schmitz, K., Catellier, D. J., McMurray, R. G., Murray, D. M., Almeida, M. J., et al. (2004b). Defining accelerometer thresholds for activity intensities in adolescent girls. Medicine and Science in Sports and Exercise, 36, 1259-1266.

Vanhelst, J., Zunquin, G., Theunynck, D., Mikulovic, J., Bui-Xuan, G., \& Béghin, L. (2009). Equivalence of accelerometer data for walking and running: Treadmill versus on land. Journal of Sports Sciences, 27(7), 669-675.

Vanhelst J., Béghin, L., Rasoamanana, P., Theunynck, D., Meskini, T., lliescu, C., Duhamel, A., Turck, D., Gottrand, F. (2010). Calibration of the RT3 accelerometer for various patterns of physical activity in children and adolescents. Journal of Sports Sciences, 28(4), 381-387.

Vanhelst J., Béghin, L., Turck, D., Gottrand, F. (2011). New validated thresholds for various intensities of physical activity in adolescents using the Actigraph accelerometer. International Journal of Rehabilitation Research, 34(2), 175-177.

Vanhelst, J., Beghin, L., Fardy, P., Bui-Xuân, G., Mikulovic, J. (2012a). A conative educational model for an intervention program in obese youth. BMC Public health, 12, 416.

Vanhelst, J., Beghin, L., Duhamel, A., et al. (2012b). Comparison of uniaxial and triaxial accelerometry in the assessment of physical activity among adolescents under free-living conditions: the HELENA study. BMC Medical Research Methodology, 12-26.

Vanhelst J., Mikulovic J., Dieu O, Blondeau T., Bui-Xuan, G., Béghin, L. (2012c). Comparison of two Actigraph accelerometer generations in the assessment of time spent in free living conditions. BMC Research Notes, 5(1), 187.

Vanhelst J., Gottrand, F., Baquet, G., Béghin, L. (2012d). Compative interinstrument reliability of uniaxial and triaxial accelerometers in free living conditions. Perceptual and Motor Skills, 114(2), 584-594.

Vigarello, G. \&, Guiet-Silvain, J. (2011). Obésité infantile, nouvelle épidémie, nouvelles interrogations. Les carrefours de l'éducation, 32, 129-132. 